\title{
Completeness for the Classical Antecedent Fragment of Inquisitive First-Order Logic
}

\section{Gianluca Grilletti ${ }^{1}$ (10)}

Accepted: 20 August 2021 / Published online: 07 October 2021

(c) The Author(s) 2021, corrected publication 2022

\begin{abstract}
Inquisitive first order logic InqBQ is an extension of first order classical logic, introducing questions and studying the logical relations between questions and quantifiers. It is not known whether InqBQ is recursively axiomatizable, even though an axiomatization has been found for fragments of the logic (Ciardelli, 2016). In this paper we define the ClAnt — classical antecedent—fragment, together with an axiomatization and a proof of its strong completeness. This result extends the ones presented in the literature and introduces a new approach to study the axiomatization problem for fragments of the logic.
\end{abstract}

Keywords Inquisitive logic $\cdot$ Completeness $\cdot$ Canonical model $\cdot$ Fragment

\section{Introduction}

Inquisitive semantics (Ciardelli et al. 2018) is a semantic framework that aims to represent statements and questions uniformly in a logical system and to analyze logical relations between them. Inquisitive first order logic InqBQ is the inquisitive counterpart of classical first order logic. In particular, it extends the usual first order language by introducing question-forming operators to represent alternative questions (e.g.,

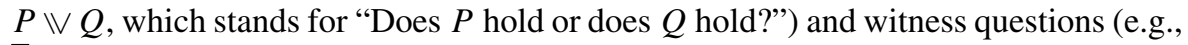
$\bar{\exists} x . P(x)$, which stands for "What is an element with property $P$ ?").

This augmented language captures concepts specific to questions-such as answerhood and dependency between questions - through the entailment of the logic; and

The author gratefully acknowledges funding from the European Research Council (ERC, grant agreement number 680220). The author would also like to thank Ivano Ciardelli, Thom van Gessel and Floris

Roelofsen for useful discussion on the manuscript and the anonymous reviewers for their comments and suggestions.

\footnotetext{
$凶$ Gianluca Grilletti

grilletti.gianluca@gmail.com

1 Munich Center for Mathematical Philosophy, Munich, Germany
} 
it allows to study the complexity of these concepts from a formal point of view: for example, is the dependency between questions recursively enumerable or compact? An effective way to tackle these questions is to characterize the entailment in terms of some fundamental principles and rules, that is, to axiomatize the logic.

Although a recursive axiomatization has been found for several propositional inquisitive logics (Ciardelli 2014; Ciardelli et al. 2020; Ciardelli and Roelofsen 2011; Punčochář 2015, 2019; Katsuhiko 2011), it is still not known whether InqBQ admits one. A sound natural deduction system for it has been proposed in Ciardelli (2016, Ch. 4), together with a conjecture of its completeness, which remains open. Ciardelli (2016, Ch. 4) it is also shown that two fragments of the logic-the mention-some fragment $\mathcal{L}_{\bar{\Xi}}$ and the mention-all fragment $\mathcal{L}_{\forall}$ —can be recursively axiomatized. This leads to the natural question whether there are other interesting fragments which are axiomatizable, and whether we can find general techniques to axiomatize them. This paper introduces the classical antecedent fragment $\mathrm{ClAnt}$, which extends $\mathcal{L}_{\forall}$ and $\mathcal{L}_{\bar{\Xi}}$, and a new approach to the completeness problem applicable to CIAnt, giving a positive answer to the first question and insights on the second.

ClAnt can be intuitively characterized as the fragment where questions are not allowed in the antecedent of an implication. This fragment is particularly interesting since it contains - modulo logical equivalence-all formulas corresponding to natural language statements and several classes of formulas corresponding to natural language questions: for example polar questions ("Will Joey come to the party?"), alternative questions ("Is Joey coming to the party or is Chandler coming?"), mention-some and mention-all questions ("What is an instance of a person coming to the party?", "Who is coming to the party?"), and their conditional versions ("If Chandler comes to the party, will Joey come to the party too?"). We prove that the natural deduction system proposed for InqBQ in Ciardelli (2016), restricted to ClAnt, provides a sound and strongly complete axiomatization of InqBQ validities in the fragment.

The paper is organized as follows: In Sect. 2 we present InqBQ and some basic properties we will use throughout the paper; in Sect. 3 we introduce the CIAnt fragment of the logic; in Sect. 4 we study a deductive system suitable to capture entailment between CIAnt formulas and show some of its main properties; Sect. 5 is devoted to showing the main result of the paper, that is, the completeness of the deductive system introduced. We conclude with some remarks and future research directions in Sect. 7.

\section{Preliminaries}

In this section we briefly present first order inquisitive logic (InqBQ) and state the results we will use in this paper. An extensive introduction on the topic is presented in Ciardelli (2016, Ch. 4). 
We start by fixing some notational conventions. With a first order signature we will refer to a finite or countable set ${ }^{1}$

$$
\Sigma=\left\{R_{0}, R_{1}, \ldots f_{0}, f_{1}, \ldots\right\}
$$

where $R_{0}, R_{1}, \ldots$ and $f_{0}, f_{1}, \ldots$ are formal symbols, referred to as relation symbols and function symbols respectively. Each symbol comes with an assigned arity, that is, the number of arguments of its interpretation in a model. We will typically use the symbols $P, Q, P^{\prime}, P_{1} \ldots$ for relation symbols of arity 1 (also called unary relation symbols), and the symbols $c, c^{\prime}, c_{1}$..for function symbols of arity 0 (also called constants).

We fix a countable set of formal symbols Var, simply referred to as the variables. We will typically use the symbols $x, y, z, x^{\prime}, x_{1} \ldots$ to indicate elements of Var. With terms of the signature $\Sigma$ (or simply terms when $\Sigma$ is clear from the context) we refer to the standard inductively defined notion of first order term: either a constant in $\Sigma$, a variable in Var or a formal combination $f\left(t_{1}, \ldots, t_{n}\right)$ where $f$ is a function symbol of arity $n$ and $t_{1}, \ldots, t_{n}$ are terms previously defined. We will typically use the symbols $t, t^{\prime}, t_{1} \ldots$ to indicate terms. Closed terms (i.e., terms not containing occurrences of variables) will play a special role in the rest of the paper: We indicate with $\mathcal{T}_{\Sigma}$ the set of closed terms of the signature $\Sigma$.

We are now ready to introduce InqBQ. The syntax of the logic is obtained by augmenting the syntax of first order logic with additional operators, i.e. inquisitive disjunction $\mathbb{V}$ and inquisitive existential quantifier $\bar{\exists}$.

Definition 1 (Syntax of InqBQ) Fix a first order signature $\Sigma$. Formulas of InqBQ are generated by the following grammar:

$$
\varphi::=\perp\left|R\left(t_{1}, \ldots, t_{n}\right)\right| \varphi \wedge \varphi|\varphi \mathbb{\vee} \varphi| \varphi \rightarrow \varphi|\forall x . \varphi| \bar{\exists} x . \varphi
$$

where $R$ is a relation symbol from $\Sigma$ and $t_{1}, \ldots, t_{n}$ are terms of the signature $\Sigma$. The concepts of free and bound occurrences of a variable, of closed formula, etc. are analogous to those of first order logic. Notice that here we are not considering the equality symbol as part of the syntax; for a treatment of the language with equality, see Sect. 6.

We introduce the following shorthands: ${ }^{2}$

$$
\neg \varphi:=\varphi \rightarrow \perp \quad \varphi \vee \psi:=\neg(\neg \varphi \wedge \neg \psi) \quad \exists x . \varphi:=\neg \forall x . \neg \varphi \quad ? \varphi:=\varphi \mathbb{\vee} \neg \varphi
$$

As noticed before, the syntax of InqBQ is an extension of the syntax of first order logic. This suggests the following definition.

\footnotetext{
1 The assumption of working with at most countable symbols is implicitly used in the proofs of Lemmas 30 and 33, so it is not dispensable in the current version of the manuscript. However, we strongly believe that these proofs can be adapted to arbitrary signatures.

2 The reader might wonder why we do not introduce a symbol $\bar{\forall}$ dual to the quantifier $\bar{\exists}$, that is, defined by the shorthand $\bar{\forall} x . \varphi:=\neg \bar{\exists} x . \neg \varphi$. There are two main reasons. Firstly, the formulas $\forall x . \neg \neg \varphi$ and $\neg \bar{\exists} x . \neg \varphi$ are provably equivalent in InqBQ, thus making the addition of $\bar{\forall}$ to the system redundant. Secondly, the formula $\bar{\forall} x . \varphi$ so defined is always equivalent to a classical formula (Definition 2), and thus $\bar{\forall}$ would not play the role of a question-forming operator as the notation would suggest.
} 
Definition 2 (Classical formula) A classical formula is a formula not containing the symbols $\mathbb{V}$ and $\bar{\exists}$.

We will usually refer to classical formulas with the symbols $\alpha, \beta, \gamma$-whereas we will use $\varphi, \psi, \chi$ for arbitrary formulas. Notice that if $\alpha$ and $\beta$ are classical formulas, then so are $\neg \alpha, \alpha \vee \beta$ and $\exists x . \alpha$.

There are two motivations to adopt this evocative naming convention: firstly, entailment between classical formulas in InqBQ coincides with first order logic entailment (this will be clarified by Lemma 10); secondly, the natural language interpretation of classical formulas is the same as the conventional one for their first order counterparts. For instance the statement "If Ross is coming to the party, then everyone is coming too" is represented by the classical formula $C(r) \rightarrow \forall x . C(x)$.

The new operators $\mathbb{V}$ and $\bar{\exists}$ are used to introduce questions and logical relations between them into the scope of the system. Inquisitive disjunction introduces alternative questions into the picture, such as "Will Joey come to the party?", corresponding to the formula ?C $(j)$; while inquisitive existential quantifier is used to introduce witness questions, such as "What is an instance of a person coming to the party?" corresponding to the formula $\bar{\exists} x . C(x)$. A more detailed account of this intuition is given in Ciardelli (2016, Ch. 4).

Since we shifted our attention from statements to sentences, we need to generalize the semantics of classical first order logic also to encompass questions. The approach adopted in InqBQ is to move from a truth-based account to an information-based one, as the latter allows us to study logical relations between statements and questions in a uniform way. To do so, we need to introduce a suitable concept of model to represent information.

Definition 3 (Information model) An information model $\mathcal{M}$ is a tuple

$$
\mathcal{M}=\left\langle M_{w} \mid w \in W\right\rangle
$$

where $W$ is a set—called the set of worlds of $\mathcal{M}$-and $M_{w}$ are first order models ${ }^{3}$ over the signature $\Sigma$ such that:

- The models $M_{w}$ (with $w \in W$ ) have the same domain $D$-called the domain of $\mathcal{M}$

- The interpretation of each function symbol $f$ of arity $n$ is the same function $f^{\mathcal{M}}: D^{n} \rightarrow D$ for each $M_{w}$ (with $w \in W$ ). This includes the interpretation of constant symbols, that is, functions of arity 0 .

For a relation symbol $R$, we indicate with $R_{w}$ the interpretation of $R$ in the first order model $M_{w}$.

We call a function $g: \operatorname{Var} \rightarrow D$ an assignment over the domain $D$. As a shorthand, we write $g[x \mapsto d]$ for the assignment that maps the variable $x \in \operatorname{Var}$ to the element $d \in D$, and otherwise coincides with $g$.

Given a term $t$ (possibly containing free variables), we indicate with $t^{\langle\mathcal{M}, g\rangle}$ the element corresponding to $t$, computed recursively in a standard way-notice that

\footnotetext{
3 In this context, a first-order model consists of a domain $D$ and an interpretation of each relation (resp. function) symbol of $\Sigma$ as a relation (resp. function) symbol of $\Sigma$ of the corresponding arity over $D$.
} 
$t^{\langle\mathcal{M}, g\rangle}$ is well defined, since the valuation of constant and function symbols is the same for every model $M_{w}$ within the information model $\mathcal{M}$. If the term $t$ is closed (i.e., if $t \in \mathcal{T}_{\Sigma}$ ) its interpretation is independent from $g$, and so we will simply write $t^{\mathcal{M}}$ omitting the assignment.

If not otherwise specified, we will indicate with $W$ and $D$ the set of worlds and the domain of the model under consideration respectively.

Definition 4 Given an information model $\mathcal{M}$ as above, we call a set $s \subseteq W$ an information state.

Information models are used to represent pieces of information. In this context, we use the term piece of information to refer to any property $I$ of first-order models in the given signature. For example $I_{1}$ : "the interpretation of $c$ is in the extension of $P$ " and $I_{2}$ : "the cardinality of the domain is finite and even" are considered pieces of information. Given a model $\mathcal{M}$ we can encode a piece of information $I$ with the info state $s_{I}:=\left\{w \in W \mid M_{w}\right.$ has property $\left.I\right\}$; that is, by selecting the worlds corresponding to the first-order models having property $I$. So in the examples given above, $s_{I_{1}}$ consists of all the worlds $w \in W$ for which $M_{w}$ satisfies $P(c)$; and $s_{I_{2}}$ is either $W$ or $\emptyset$, depending on the cardinality of $D$.

We are now ready to present the semantics of InqBQ.

Definition 5 (Semantics of InqBQ) Let $\mathcal{M}$ be an information model, $s$ an information state of $\mathcal{M}$ and $g$ : Var $\rightarrow D$ an assignment. We define the support relation $\vDash$ over formulas of InqBQ by the following inductive clauses:

$$
\begin{array}{ll}
\mathcal{M}, s \vDash_{g} \perp & \Longleftrightarrow s=\emptyset \\
\mathcal{M}, s \vDash_{g} R\left(t_{1}, \ldots, t_{n}\right) & \Longleftrightarrow \text { For all } w \in s \text { it holds } R_{w}\left(t_{1}^{\langle\mathcal{M}, g\rangle}, \ldots, t_{n}^{\langle\mathcal{M}, g\rangle}\right) \\
\mathcal{M}, s \vDash_{g} \psi_{1} \wedge \psi_{2} & \Longleftrightarrow \mathcal{M}, s \vDash_{g} \psi_{1} \text { and } \mathcal{M}, s \vDash_{g} \psi_{2} \\
\mathcal{M}, s \vDash_{g} \psi_{1} \mathbb{\vee} \psi_{2} & \Longleftrightarrow \mathcal{M}, s \vDash_{g} \psi_{1} \text { or } \mathcal{M}, s \vDash_{g} \psi_{2} \\
\mathcal{M}, s \vDash_{g} \psi_{1} \rightarrow \psi_{2} & \Longleftrightarrow \text { For all } s^{\prime} \subseteq s, \text { if } \mathcal{M}, s^{\prime} \vDash_{g} \psi_{1} \text { then } \mathcal{M}, s^{\prime} \vDash_{g} \psi_{2} \\
\mathcal{M}, s \vDash_{g} \forall x . \psi & \Longleftrightarrow \text { For all } d \in D \text { it holds } \mathcal{M}, s \vDash_{g[x \mapsto d]} \psi \\
\mathcal{M}, s \vDash_{g} \bar{\exists} x . \psi & \Longleftrightarrow \text { There exists } d \in D \text { such that } \mathcal{M}, s \vDash_{g[x \mapsto d]} \psi
\end{array}
$$

If $\mathcal{M}, s \vDash_{g} \varphi$ we say that $s$ supports $\varphi$ under $g$. We introduce the shorthand $\mathcal{M} \vDash_{g} \varphi$ for $\mathcal{M}, W \vDash_{g} \varphi$. It can be verified that, if $\varphi$ is a sentence, then its semantics is independent from the assignment $g$. In this case we will simply omit $g$.

In what follows, we will give a brief introduction to the support semantics, focusing on the results instrumental for the rest of the paper. For a thorough description of the support semantics, we point to Ciardelli (2016, Ch. 4). We start by stating one of the main properties of this semantics.

Lemma 1 (Downward closure and empty state property) Let $\varphi$ be a formula, $s, s^{\prime}$ information states of $\mathcal{M}$ and $g: D \rightarrow$ Var an assignment. Then:

- If $\mathcal{M}, s \vDash_{g} \varphi$ and $s^{\prime} \subseteq s$ then $\mathcal{M}, s^{\prime} \vDash_{g} \varphi$;

$-\mathcal{M}, \emptyset \vDash_{g} \varphi$. 
These two properties have an intuitive interpretation, in line with the one presented for information models: if a certain piece of information supports a sentence $\left(\mathcal{M}, s \vDash_{g} \varphi\right)$, then any more specific information supports the same sentence too (if $s^{\prime} \subseteq s$ then $\left.\mathcal{M}, s^{\prime} \vDash_{g} \varphi\right)$; and an inconsistent piece of information supports everything $\left(\mathcal{M}, \varnothing \vDash_{g}\right.$ $\varphi)$.

Using Lemma 1, we can also easily derive a semantic clause for the operator $\neg$ :

$$
\begin{aligned}
& \mathcal{M}, s \vDash_{g} \neg \varphi \\
& \Longleftrightarrow \text { For all } s^{\prime} \subseteq s, \text { if } \mathcal{M}, s^{\prime} \vDash_{g} \varphi \text { then } \mathcal{M}, s^{\prime} \vDash_{g} \perp \\
& \Longleftrightarrow \text { For all } s^{\prime} \subseteq s, \mathcal{M}, s^{\prime} \not \vDash_{g} \varphi \text { or } s^{\prime}=\emptyset \\
& \Longleftrightarrow \text { For all } w \in s, \mathcal{M},\{w\} \nvdash_{g} \varphi
\end{aligned}
$$

As usual, we associate to the semantics just introduced the corresponding concepts of validity and entailment.

Definition 6 (Validity and entailment) We say that a formula $\varphi$ is (semantically) valid in InqBQ, and indicate it with $\vDash \varphi$, iff for every model $\mathcal{M}$, every information state $s$ of $\mathcal{M}$ and every assignment $g: \operatorname{Var} \rightarrow D$, it holds $\mathcal{M}, s \vDash_{g} \varphi$.

Given a set of formulas $\Phi \cup\{\psi\}$, we say that $\Phi$ entail $\psi$, and indicate it with $\Phi \vDash \psi$, iff for every $\mathcal{M}, s$ and $g$ as above, if $\mathcal{M}, s \vDash_{g} \varphi$ for every $\varphi \in \Phi$, then $\mathcal{M}, s \vDash_{g} \psi$.

As an example, consider the formulas $P(c) \vee \neg P(c)$ and $P(c) \vee \neg P(c)$. Unraveling the semantic clauses, we obtain the following support conditions for the two formulas.

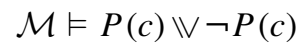

$$
\begin{aligned}
& \Longleftrightarrow \mathcal{M} \vDash P(c) \text { or } \mathcal{M} \vDash \neg P(c) \\
& \Longleftrightarrow\left[\forall w . P_{w}\left(c^{\mathcal{M}}\right) \text { holds }\right] \text { or }\left[\forall w . P_{w}\left(c^{\mathcal{M}}\right) \text { does not hold }\right] \\
& \mathcal{M} \vDash P(c) \vee \neg P(c) \\
& \Longleftrightarrow \mathcal{M} \vDash \neg(\neg P(c) \wedge \neg \neg P(c)) \\
& \Longleftrightarrow \forall w . \mathcal{M},\{w\} \not \models \neg P(c) \wedge \neg \neg P(c) \\
& \Longleftrightarrow \forall w .[\mathcal{M},\{w\} \not \models \neg P(c) \text { or } \mathcal{M},\{w\} \not \models \neg \neg P(c)] \\
& \Longleftrightarrow \forall w \cdot\left[P_{w}\left(c^{\mathcal{M}}\right) \text { holds or } \mathcal{M},\{w\} \vDash \neg P(c)\right] \\
& \Longleftrightarrow \forall w \cdot\left[P_{w}\left(c^{\mathcal{M}}\right) \text { holds or } \mathcal{M},\{w\} \not \models P(c)\right] \\
& \Longleftrightarrow \forall w \cdot\left[P_{w}\left(c^{\mathcal{M}}\right) \text { holds or } P_{w}\left(c^{\mathcal{M}}\right) \text { does not hold }\right]
\end{aligned}
$$

It is clear that the second condition is always true, since in every world the extension of $P$ is determined: that is, $P(c) \vee \neg P(c)$ is valid in InqBQ. However, the first condition is not tautological: It requires all worlds in $\mathcal{M}$ to agree on whether $c$ is in the extension of $P$. 
We observe a similar situation with the formulas $\bar{\exists} x . P(x)$ and $\exists x . P(x)$, for which the support conditions can be derived with a similar proof.

$$
\begin{aligned}
& \mathcal{M} \vDash \bar{\exists} x \cdot P(x) \Longleftrightarrow \text { there is } d \in D \text { such that for all } w \in W, P_{w}(d) \text { holds } \\
& \mathcal{M} \vDash \exists x \cdot P(x) \Longleftrightarrow \text { for all } w \in W, \text { there is } d_{w} \in D \text { such that } P_{w}\left(d_{w}\right) \text { holds }
\end{aligned}
$$

In this case, $\exists x . P(x)$ requires that in every world there is an element with property $P$-possibly depending on the world; while the formula $\bar{\exists} x . P(x)$ asks more, namely that for a fixed element $d$, all the worlds agree on $d$ being in the extension of $P$. So the additional requirement is that we have a witness for the quantifier which all the worlds agree on.

The differences between the operators $\vee$ and $\vee$, and between the operators $\bar{\exists}$ and $\exists$ are also witnessed by two peculiar properties of the support semantics: the disjunction and existence properties for the inquisitive operators.

Theorem 7 (Theorems 4.1 and 4.2 in Grilletti (2019)) Fix a signature $\Sigma$. Given a set of classical formulas $\Gamma$, for every formulas $\varphi$ and $\psi$ we have:

- If $\Gamma \vDash \varphi \mathbb{\Downarrow} \psi$, then $\Gamma \vDash \varphi$ or $\Gamma \vDash \psi$;

- If $\Gamma \vDash \bar{\exists} x . \varphi$, then $\Gamma \vDash \varphi[t / x]$ for some term $t$ of $\Sigma$.

In particular, this highlights the constructive character of the inquisitive operators $\mathbb{V}$ and $\bar{\exists}$, in contrast with the operators $\vee$ and $\exists$ which behave classically.

However, if we restrict our attention to singleton information states, that is, information states containing only one world, we can see that the support semantics is a generalization of the usual semantics of first order classical logic. In this case the support condition for $\$ and $\vee$ coincide, the support condition for $\exists$ and $\bar{\exists}$ also coincide, and so a formula $\varphi$ is supported in a state $\{w\}$ if and only if its classical variant $\varphi^{c l}$ —obtained by substituting $\vee$ for $\mathbb{\vee}$ and $\exists$ for $\bar{\exists}$ in $\varphi$-is true in $M_{w}$.

The following result generalizes the previous observation and connects classical semantics and support semantics. We will indicate with $\models^{c l}$ both the standard semantics relation of classical first order logic and the corresponding entailment relation between classical formulas.

Lemma 8 Let $\alpha$ be a classical formula. Then

$$
\mathcal{M}, s \vDash_{g} \alpha \quad \Longleftrightarrow \quad \forall w \in s . \mathcal{M},\{w\} \vDash_{g} \alpha \quad \Longleftrightarrow \quad \forall w \in s . M_{w} \vDash_{g}^{c l} \alpha
$$

In particular, a classical formula is valid for support semantics iff it is valid for the classical semantics; and the entailment relations $\vDash$ and $\vDash^{c l}$ coincide over classical formulas.

With a slight abuse of notation (justified by the lemma), henceforth we will use the symbol $\vDash$ both for support semantics (resp., entailment) and for the standard semantics (resp., entailment) of classical first order logic, omitting the superscript $\mathrm{cl}$.

This result has rather interesting consequences and will be used several times in the current manuscript. For example we have the following: given a classical formula $\alpha$, a model $\mathcal{M}$ and an assignment $g$, there is always a greatest information state satisfying 
$\alpha$, namely the set $|\alpha|:=\left\{w \in W \mid M_{w} \vDash_{g}^{c l} \alpha\right\}$; and given an information state $s$, the state $s \cap|\alpha|$ is the greatest substate of $s$ supporting $\alpha$.

Lemma 8 is particularly interesting under the information-based interpretation: given an information state $s$, a certain statement-encoded by $\alpha$-is supported by $s$ if and only if in every possible world in $s$ the statement is true. Moreover, under this interpretation $|\alpha|$ represents exactly the information conveyed by the statement encoded by $\alpha$.

Similarly, we can also talk about support for questions: a question is supported by an information state $s$ iff $s$ resolves the issue raised by the question. So for example $C(j) \Vdash \neg C(j)$, representing the alternative question "Will Joey come to the party or not?", is supported by $s$ iff in all the possible worlds Joey will go to the party $o r$ in all the possible worlds Joey will not go to the party; that is, the information encoded by $s$ must be enough to determine an answer to the question. For an exhaustive treatment of the conceptual background of the logic we refer to Ciardelli (2016, Chs. 1, 2 and 4).

We conclude this section by presenting a sound natural deduction system for InqBQ and some related concepts.

Definition 9 (Natural deduction system) Consider the natural deduction system in Table 1 . We say that a set of formulas $\Phi$ derives a formula $\psi$ (in symbols $\Phi \vdash \psi$ ) if there is a derivation of $\psi$ from $\Phi$ in the natural deduction system.

This system was presented in Ciardelli (2016, Ch. 4). It is still an open question if the system is complete for InqBQ. What is known Ciardelli (2016, Proposition 4.4.3) is that this system is complete for classical consequences. Given a set of formulas $\Phi$, define $\Phi^{c l}:=\left\{\varphi^{c l} \mid \varphi \in \Phi\right\}$. We will indicate with $\left.\right|^{c l}$ the standard provability relation of classical first order logic.

Lemma 10 Let $\Phi$ be a set of inquisitive formulas and $\alpha$ a classical formula. Then

$$
\left.\Phi \vdash \alpha \Longleftrightarrow \Phi^{c l} \vdash \alpha \Longleftrightarrow \Phi^{c l}\right|^{c l} \alpha
$$

In particular, $\vdash$ coincides with the usual classical entailment when $\Phi$ consists only of classical formulas; and a classical formula is valid in InqBQ if and only if it is valid in classical first order logic.

With slight abuse of notation (justified by the lemma), we will indicate with $\vdash$ both the provability relation from Definition 9 and the standard provability relation of classical first order logic, omitting the superscript $c l$.

It is worth discussing two rules of this system, which play an essential role in the rest of the paper: the $\mathbb{V}$-split rule and the $\bar{\exists}$-split rule. Notice that these rules are natural counterparts of the disjunction and existence properties of the support semantics. Moreover, these rules have a natural reading under the information-based interpretation: if a statement resolves an alternative question $(\alpha \rightarrow \psi \vee \chi)$, then it supports one of the alternatives $((\alpha \rightarrow \psi) \vee(\alpha \rightarrow \chi))$; and if a statement resolves a witness question $(\alpha \rightarrow \bar{\exists} x \cdot \psi)$, then it provides enough information to pinpoint a witness $(\bar{\exists} x \cdot(\alpha \rightarrow \psi))$. 
Table 1 Natural deduction system for InqBQ

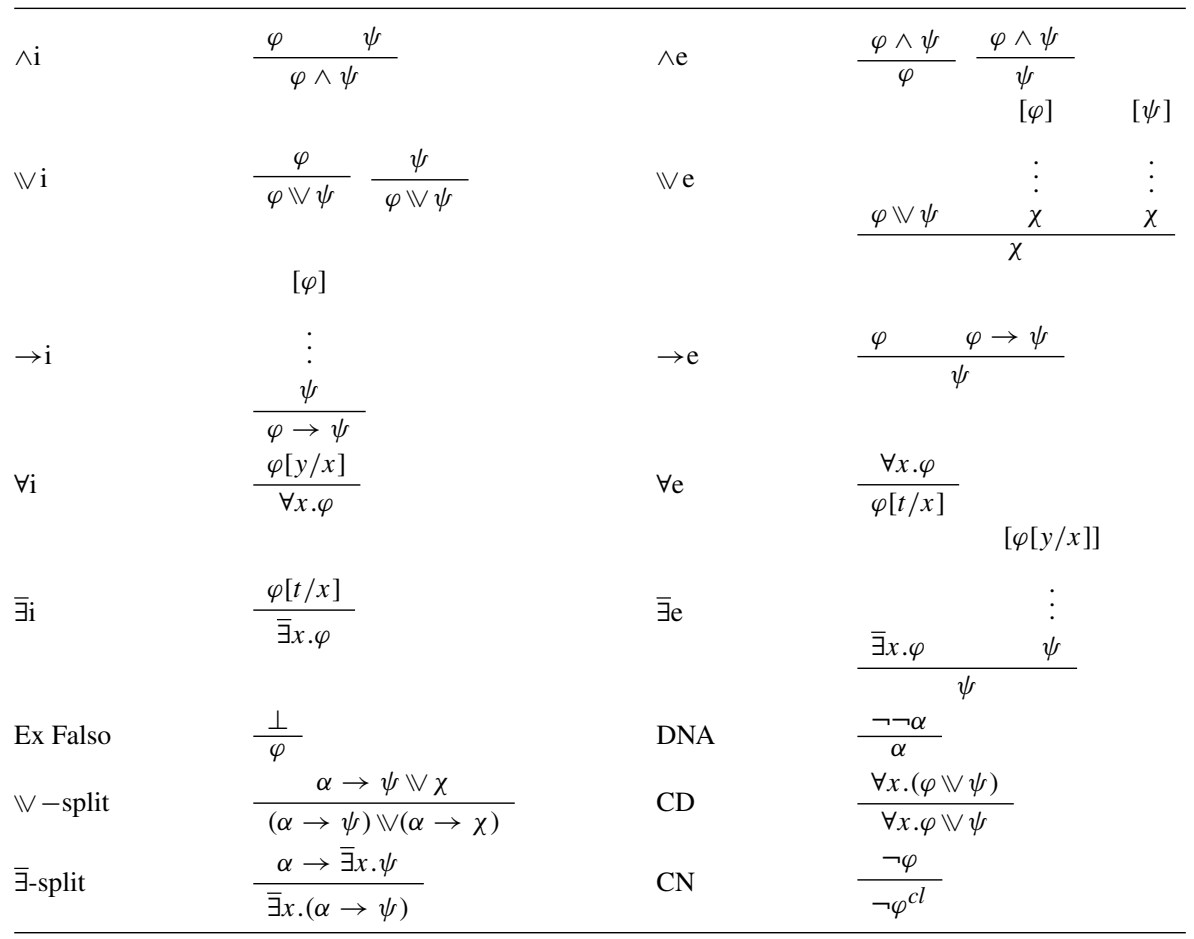

In $(\forall e)$ and $(\bar{\exists} i), t$ must be free for $x$ in $\varphi$; in $(\forall i), y$ must not occur free in any undischarged assumption; in $(\bar{\exists} e), y$ must not occur free in $\psi$ or any undischarged assumption; in $(D N A), \alpha$ ranges over classical formulas; in ( $\vee$-split), $\alpha$ ranges over classical formulas; in ( $\bar{\exists}$-split), $\alpha$ ranges over classical formulas and $x$ is not free in $\alpha$; in $(C D), x$ must not occur free in $\psi ;$ in $(C N), \varphi^{c l}$ stands for the classical variant of $\varphi$

The proofs of soundness of the rules follow this same intuition. Let us show the case of $\mathbb{V}$-split as an example:

$$
\begin{aligned}
& \mathcal{M}, s \vDash_{g} \alpha \rightarrow \psi \mathbb{\vee} \chi \\
\Longleftrightarrow & \text { For all } s^{\prime} \subseteq s, \text { if } \mathcal{M}, s^{\prime} \vDash_{g} \alpha \text { then } \mathcal{M}, s^{\prime} \vDash_{g} \psi \mathbb{V} \chi \\
\Longleftrightarrow & \mathcal{M}, s \cap|\alpha| \vDash_{g} \psi \mathbb{\vee} \chi \\
\Longleftrightarrow & \mathcal{M}, s \cap|\alpha| \vDash_{g} \psi \text { or } \mathcal{M}, s \cap|\alpha| \vDash_{g} \chi \\
\Longleftrightarrow & \mathcal{M}, s \vDash_{g} \alpha \rightarrow \psi \text { or } \mathcal{M}, s \vDash_{g} \alpha \rightarrow \chi \\
\Longleftrightarrow & \mathcal{M}, s \vDash_{g}(\alpha \rightarrow \psi) \mathbb{\vee}(\alpha \rightarrow \chi)
\end{aligned}
$$

by Lemma 1

by Lemma 1

Finally, notice that the rules are not sound without the restriction on the antecedent $\alpha$ being classical. For example, consider the formula

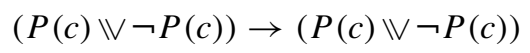


which is trivially valid. If we could apply the rule to this formula, by the disjunction property (Theorem 7) we would have that $(P(c) \Vdash \neg P(c)) \rightarrow P(c)$ would be valid

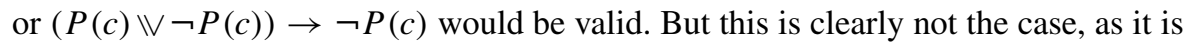
easy to produce counterexamples for both formulas.

\section{ClAnt Fragment}

In this section we present the main protagonist of this paper, that is, the CIAnt fragment.

Definition 11 (ClAnt fragment) The ClAnt fragment is generated by the following grammar:

$$
\varphi::=\perp\left|R\left(t_{1}, \ldots, t_{n}\right)\right| \varphi \wedge \varphi|\varphi \mathbb{V} \varphi| \alpha \rightarrow \varphi|\forall x . \varphi| \bar{\exists} x . \varphi
$$

where $\alpha$ ranges over classical formulas.

In Ciardelli (2016, Ch. 4), two other fragments were presented and studied, namely the mention-some $\left(\mathcal{L}_{\bar{\exists}}\right)$ and the mention-all fragment $\left(\mathcal{L}_{\forall}\right)$. These two fragments are generated by the following grammars:

$$
\begin{aligned}
& \mathcal{L}_{\bar{\Xi}} \quad \text { Mention-some: } \quad \varphi::=\alpha|\varphi \mathbb{V} \varphi| \bar{\exists} x . \varphi|\varphi \wedge \varphi| \alpha \rightarrow \varphi \\
& \mathcal{L}_{\forall} \text { Mention-all: } \quad \varphi::=\alpha|? \alpha| \forall x . \varphi
\end{aligned}
$$

where $\alpha$ ranges over classical formulas.

It was proven in Ciardelli (2016) that the support relation for inquisitive logic restricted to both these fragments is axiomatizable. Interestingly, the proofs presented are quite different and cannot, prima facie, be adapted to the other fragment. For the mention-some fragment, the completeness proof uses a canonical model construction similar to the one proposed for propositional inquisitive logic in Ciardelli (2016, Ch. 3 ), heavily relying on the existence of a disjunctive normal form for formulas. For the mention-all fragment, the completeness proof passes through a translation in the Logic of Interrogation (Groenendijk 1999; ten Cate and Shan 2007), a logic with a partition-based semantics.

Notice that CIAnt subsumes both these fragments. So the axiomatization for CIAnt and the corresponding completeness proof, presented in Sects. 4 and 5 respectively, introduce a novel approach to axiomatize both $\mathcal{L}_{\bar{\Xi}}$ and $\mathcal{L}_{\forall}$. Moreover, CIAnt is strictly more expressive than both these fragments, as shown by the following result.

Proposition 12 The sentence $\forall x . \bar{\exists} y .(P(x) \leftrightarrow \neg P(y))$ is in CIAnt and it is not logically equivalent to any formula in $\mathcal{L}_{\bar{\exists}} \cup \mathcal{L}_{\forall}$ in the same signature.

This formula holds if for every element $x$ there is an associated element $y$ such that exactly one of them has property $P$. This condition is particularly interesting in contexts where epistemic identity does not correspond to ontological identity, like inquisitive logic (see Ciardelli 2016, Sec. 4.3.4 for a small discussion on the topic). 


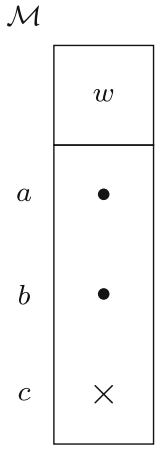

(a) Two inquisitive models in the signature $\Sigma=\{P\}$, with $P$ a unary symbol. The top row represents the set of worlds of the model (e.g., $\{w, v\}$ for the model $\mathcal{N})$ and the leftmost column represents the domain ( $\{a, b, c\}$ for both models); the extension of $P$ at each world is encoded by the entries of the table: a $\bullet$ indicates that the element corresponding to the row is in the extension of $P$ in the world corresponding to the column, while a $\times$ indicates it is not (e.g., $a \in P_{w}^{\mathcal{M}}$ in and $\left.b \notin P_{v}^{\mathcal{N}}\right)$.
$\mathcal{N}$

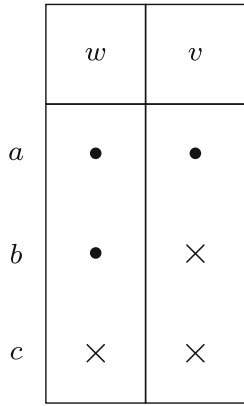

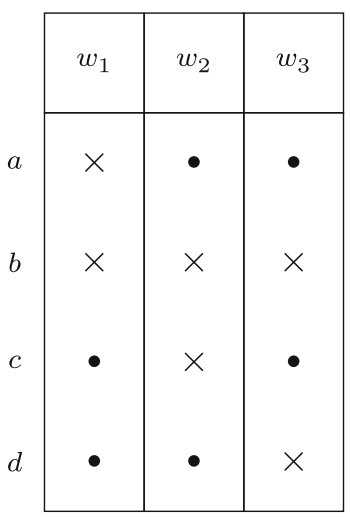

(b) A model in the signature $\{P\}$ for $P$ a unary predicate. We use here the same representation described in Figure 1a.

Fig. 1 The models used in the proof of Proposition 12

Proof In the scope of this proof, we will use the notation $\theta:=\forall x \cdot \bar{\exists} y \cdot(P(x) \leftrightarrow$ $\neg P(y)) . \theta$ is clearly in ClAnt-notice that $P(x) \leftrightarrow \neg P(y)$ is a classical formula.

To show that $\theta$ is not equivalent to a formula in $\mathcal{L}_{\bar{\Xi}}$, consider the models depicted in Fig. 1a. It is straightforward to verify that $\mathcal{M} \vDash \theta$, while $\mathcal{N} \not \models \theta$. Assume towards a contradiction that $\theta$ is equivalent to a formula in $\mathcal{L}_{\bar{\Xi}}$. By the normal form described in Ciardelli (2016, Proposition 4.7.2), this means that

$$
\theta \equiv \bar{\exists} \bar{x}_{1} \cdot \alpha_{1} \vee \ldots \mathbb{\vee} \bar{\exists} \bar{x}_{n} \cdot \alpha_{n}
$$

for some $\alpha_{1}, \ldots, \alpha_{n}$ classical formulas. In particular, this means that for some $i \in$ $\{1, \ldots, n\}$ we have $\mathcal{M} \vDash \bar{\exists} \bar{x}_{i} . \alpha_{i}$, that is, $\mathcal{M} \vDash_{g} \alpha_{i}$ for some assignment $g$.

Notice that the only atomic formulas available in the current language are $\perp$ and $P(x)$ for $x$ a variable. So if $g(x)=b$, the assignments $g$ and $g[x \mapsto a]$ satisfy exactly the same atomic formulas, and consequently the same complex formulas. And the same applies for $g$ and the assignment $h$ defined as follows:

$$
h(x)= \begin{cases}g(x) & \text { if } g(x) \in\{a, c\} \\ a & \text { if } g(x)=b\end{cases}
$$

Since the image of $h$ is contained in $\{a, c\}$ and $\mathcal{M} \vDash_{h} \alpha_{i}$, with a similar reasoning we also obtain that $\mathcal{N},\{w\} \vDash_{h} \alpha_{i}$ and $\mathcal{N},\{v\} \vDash_{h} \alpha_{i}$. Thus by Lemma 8 we have $\mathcal{N} \vDash_{h} \alpha_{i}$ and consequently $\mathcal{N} \vDash \theta$. And this is a contradiction, as wanted. 
To show that $\theta$ is not equivalent to a formula in $\mathcal{L}_{\forall}$ we use Ciardelli (2016, Proposition 4.8.4), which states that every formula $\varphi \in \mathcal{L}_{\forall}$ is pair-distributive, that is:

$$
\mathcal{M}, s \vDash_{g} \varphi \quad \text { iff } \quad \forall s^{\prime} \subseteq s .\left[\left|s^{\prime}\right| \leq 2 \Longrightarrow \mathcal{M}, s^{\prime} \vDash_{g} \varphi\right]
$$

So we just need to show that $\theta$ is not pair-distributive: given the model in Fig. 1b, every state $s^{\prime}$ with at most two worlds satisfies $\theta$, but the whole model does not.

We conclude this section with an alternative presentation of the CIAnt fragment.

Lemma 13 Every formula in CIAnt is equivalent to a formula generated by the following grammar:

$$
\varphi::=\alpha|\varphi \wedge \varphi| \varphi \mathbb{\vee} \varphi|\forall x . \varphi| \bar{\exists} x . \varphi
$$

where $\alpha$ ranges over classical formulas.

Proof (Sketch) The main idea of the proof is to "massage" the implications toward the classical formulas using the following equivalences, taking care of renaming the bounded variables when necessary.

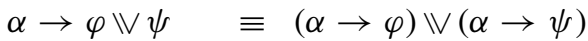

$$
\begin{aligned}
& \alpha \rightarrow \varphi \wedge \psi \quad \equiv(\alpha \rightarrow \varphi) \wedge(\alpha \rightarrow \psi) \\
& \alpha \rightarrow(\beta \rightarrow \varphi) \equiv(\alpha \wedge \beta) \rightarrow \varphi \\
& \alpha \rightarrow \forall x . \varphi \quad \equiv \forall x .(\alpha \rightarrow \varphi) \\
& \alpha \rightarrow \bar{\exists} x \cdot \varphi \quad \equiv \bar{\exists} x \cdot(\alpha \rightarrow \varphi) \\
& \text { (For } x \text { not free in } \alpha \text { ) } \\
& \text { (For } x \text { not free in } \alpha \text { ) }
\end{aligned}
$$

Notice that the equivalences read from left to right reduce the complexity of the consequent of the implication. Consequently, after enough reduction steps all the implications appear in subformulas of the form $\alpha \rightarrow \beta$, which are themselves classical formulas.

This result tells us that we can dispense with implications outside of classical formulas. At the level of expressive power this is a significant limitation, since $\rightarrow$ is the only logical operator acting as a second-order quantifier for the semantics-compare with Definition 5. It is not clear yet whether the completeness proof presented in the following sections relies on this limitation or can be generalized to more expressive fragments, or even the whole logic. What is known, is that CIAnt is strictly less expressive than InqBQ, as the following result shows.

Claim The formula $\forall x . ? P(x) \rightarrow$ ? $r$ (for $P$ a unary relation symbol and $r$ a 0 -ary relation symbol) is not logically equivalent to any formula in CIAnt.

The only proof known to the author of this result relies on Lemma 13, but uses also a variation of the Ehrenfeucht-Fraïssé game for InqBQ introduced in Gianluca and Ivano (2019), which has not yet been discussed in the literature. Since presenting and discussing these results strides away from the intended objective of this paper, we leave the claim unproven for now, with the promise to present the proof in future works. 


\section{Deductive System}

Definition 14 (Natural deduction system for CIAnt) For $\Phi \cup\{\psi\}$ CIAnt formulas we say that $\Phi$ derives $\psi$ in CIAnt (in symbols $\Phi \vdash \psi$ ) if there is a derivation of $\psi$ from $\Phi$ containing only CIAnt formulas.

Clearly if $\Phi \vdash \psi$ then $\Phi \vdash \psi$, but a priori nothing ensures that if there exists a derivation of $\psi$ from $\Phi$, then there exists also a derivation containing only CIAnt formulas. However, we will show in Theorem 34 that this is indeed the case.

Notice that if we apply a rule in Table 1 to CIAnt formulas, the conclusion produced is again a ClAnt formula, the only exception being $(\rightarrow i)$. In particular, the conclusion of $(\rightarrow i)$ is a ClAnt formula iff the discharged assumption is a classical formula, that is, if the rule is applied with the following side condition:

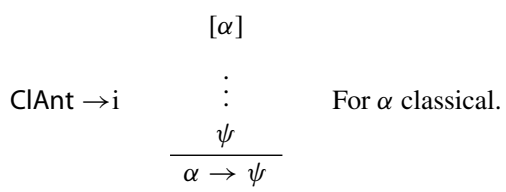

From this observation, it follows that $\sim$ can be characterized as the provability relation of the natural deduction system obtained by replacing the rule $(\rightarrow \mathrm{i})$ with the rule $(\mathrm{ClAnt} \rightarrow \mathrm{i})$ in Table 1 .

To study the properties of this system and the relations with the system presented in Definition 9, we focus on theories of InqB and of CIAnt, that is, sets of formulas in InqB and in CIAnt respectively. Since we need to be particularly careful when handling free variables, we distinguish between sets of formulas and theories.

Definition 15 Let $\Sigma$ be a fixed signature.

- A set of $\Sigma$-formulas is any set $\Phi$ of InqB formulas in the signature $\Sigma$.

- A $\Sigma$-theory is any set $\Phi$ of InqB sentences in the signature $\Sigma$.

So $\Sigma$-theories do not contain formulas with free variables, while sets of $\Sigma$-formulas may. It is easy to transform a set of $\Sigma$-formulas in a corresponding theory, at the cost of adding new constant symbols to the signature. For $A$ a set of parameters-that we assume disjoint from the set $\Sigma$-we define $\Sigma(A)$ as the signature extending $\Sigma$ with the elements of $A$ as fresh constant symbols.

Definition 16 Let $\Phi$ be a set of $\Sigma$-formulas and let $V$ be the set of free variables appearing in $\Phi$. Consider a set $\widetilde{V}:=\{\tilde{x} \mid x \in V\}$ of distinct formal parameters. We define the closure of $\Phi$ as the $\Sigma(\widetilde{V})$-theory $\widetilde{\Phi}$ obtained by substituting every free occurrence of the variable $x$ in $\Phi$ with $\tilde{x}$, for every $x \in V$.

Proposition 17 Let $\Phi \cup\{\psi\}$ be a set of $\Sigma$-formulas. Then ${ }^{4}$

$$
\Phi \vDash \psi \quad \Longleftrightarrow \quad \widetilde{\Phi} \vDash \widetilde{\psi}
$$

\footnotetext{
${ }^{4}$ If the set $\Phi$ and $\psi$ have a common free variable, let us say $x, \widetilde{\Phi}$ and $\widetilde{\psi}$ are obtained by substituting the free occurrences of $x$ with the same formal parameter $\tilde{x}$.
} 
The proof consists only in comparing the semantic clauses of the two entailments, and it is therefore omitted. This proposition allows us to focus our attention on theories and to highlight the role of the parameters in the proofs that follow.

To prove the completeness of the system introduced, we need to study more in detail three special classes of theories: saturated theories, classically saturated theories and CIAnt-saturated theories. In what follows, we will indicate with $A$ a fixed set of constant symbols not appearing in the signature $\Sigma$.

Definition 18 (Saturated theory) A $\Sigma(A)$-theory $\Phi$ is called saturated (with respect to $A$ ) if for every sentences $\varphi, \psi$ of $\Sigma(A)$ it satisfies:

- Coherence: $\quad \Phi \mid+\perp$;

- Deductive closure: $\quad$ if $\Phi \vdash \varphi$ then $\varphi \in \Phi$;

- Disjunction property: If $\Phi \vdash \varphi \mathbb{\vee} \psi$ then $\Phi \vdash \varphi$ or $\psi \vdash \varphi$;

- Existence property: If $\Phi \vdash \bar{\exists} x . \varphi$ then $\Phi \vdash \varphi[t / x]$ for some $t \in \mathcal{T}_{\Sigma(A)}$;

- Normality condition: If $\Phi \forall \forall x . \varphi$ then $\Phi+\varphi \varphi[t / x]$ for some $t \in \mathcal{T}_{\Sigma(A)}$.

It is easy to produce examples of saturated theories: Consider an inquisitive model $\mathcal{M}$ on the signature $\Sigma(A)$ for which the interpretations of the terms in $\mathcal{T}_{\Sigma(A)}$ cover the whole domain, that is, $\left\{t^{\mathcal{M}} \mid t \in \mathcal{T}_{\Sigma(A)}\right\}=D$-henceforth we will call these models $\mathcal{T}_{\Sigma(A)}$-covered. Given an information state $s$, define the theory of $\langle\mathcal{M}, s\rangle$ as $\operatorname{Th}(\mathcal{M}, s)=\{\varphi$ sentence of $\Sigma(A) \mid \mathcal{M}, s \vDash \varphi\}$. It is immediate to show that $\operatorname{Th}(\mathcal{M}, s)$ is a saturated $\Sigma(A)$-theory. In particular, the existence property and the normality condition rely on the fact that the model is $\mathcal{T}_{\Sigma(A)}$-covered.

If we restrict our attention only to classical formulas, we can define the corresponding concept of classically-saturated theory.

Definition 19 (Classical theories and classically saturated theories) A classical $\Sigma$ theory is a $\Sigma$-theory containing only classical formulas.

We say that a classical $\Sigma(A)$-theory $\Gamma$ is classically-saturated (with respect to $A$ ) if for every classical sentences $\alpha, \beta$ of $\Sigma(A)$ it satisfies:

- Coherence:

$\Gamma \not+\perp$;

- Deductive closure:

If $\Gamma \vdash \alpha$, then $\alpha \in \Gamma$;

- Classical disjunction property: If $\Gamma \vdash \alpha \vee \beta$ then $\Gamma \vdash \alpha$ or $\Gamma \vdash \beta$;

- Classical existence property: If $\Gamma \vdash \exists x . \alpha$ then $\Gamma \vdash \alpha[t / x]$, for some $t \in \mathcal{T}_{\Sigma(A)}$.

A simple induction shows that, given $\Gamma \cup\{\alpha\}$ classical formulas, $\Gamma \vdash \alpha$ if and only if $\alpha$ is a consequence of $\Gamma$ in classical first order logic. This observation, in conjunction with deductive closure and the disjunction property, tells us that a classically-saturated theory $\Gamma$ is complete, that is, for every sentence $\alpha$ of $\Sigma(A)$ we have either $\alpha \in \Gamma$ or $\neg \alpha \in \Gamma$. And in turns, it follows that the condition corresponding to normality is also satisfied:

$$
\begin{aligned}
& \Gamma \nvdash \forall x . \alpha \\
& \Rightarrow \Gamma \vdash \exists x . \neg \alpha \\
& \Rightarrow \Gamma \vdash \neg \alpha[t / x], \text { for some } t \in \mathcal{T}_{\Sigma(A)} \\
& \Rightarrow \Gamma \mid+\alpha[t / x], \text { for some } t \in \mathcal{T}_{\Sigma(A)}
\end{aligned}
$$


Classically-saturated theories are examples of Hintikka sets for classical first order logic (see for example Hodges 1993, Sec. 2.3). This is particularly relevant, since every Hintikka set $\Gamma$ admits a first-order model $M_{\Gamma}$, and it is thus satisfiable.

We will sketch the construction of $M_{\Gamma}$ for $\Gamma$ a classically-saturated $\Sigma(A)$-theory, essentially following the proof of Theorem 2.3.3 in Hodges (1993).

Definition 20 Given $\Gamma$ a classically-saturated $\Sigma(A)$-theory, we define the model $M_{\Gamma}$ by the following clauses:

- The domain of $M_{\Gamma}$ is the set $\mathcal{T}_{\Sigma(A)}$;

- A constant symbol $c \in \Sigma$ is interpreted as itself, that is, $c^{M_{\Gamma}}=c$. The same applies for a parameter $a \in A: a^{M_{\Gamma}}=a$.

- An $n$-ary function symbol $f$ different from a constant is interpreted as the corresponding term combinator, that is:

$$
\begin{aligned}
& f^{M_{\Gamma}:\left(\mathcal{T}_{\Sigma(A)}\right)^{n}} \rightarrow \mathcal{T}_{\Sigma(A)} \\
& \left\langle t_{1}, \ldots, t_{n}\right\rangle \mapsto f\left(t_{1}, \ldots, t_{n}\right)
\end{aligned}
$$

- An $n$-ary relation symbol $R$ is interpreted as the relation defined by the following clause:

$$
R^{M_{\Gamma}}\left(t_{1}, \ldots, t_{n}\right) \quad \text { iff } \quad R\left(t_{1}, \ldots, t_{n}\right) \in \Gamma
$$

Notice that by construction the model $M_{\Gamma}$ is $\mathcal{T}_{\Sigma(A)}$-covered. Another peculiar property of $M_{\Gamma}$ is that it satisfies exactly the sentences in $\Gamma$, that is:

Lemma 21 (Truth Lemma) For every classical sentence $\alpha$ of $\Sigma(A)$ we have

$$
M_{\Gamma} \vDash \alpha \quad \text { iff } \quad \alpha \in \Gamma
$$

The proof of this result (which we omit) consists of a simple structural induction on $\alpha$. As an example, we sketch the inductive step for the case $\alpha$ of the form $\exists x . \beta$.

$$
\begin{array}{rlr} 
& M_{\Gamma} \vDash \exists x . \beta & \\
\Longleftrightarrow & M_{\Gamma} \vDash \beta[t / x] \text { for some } t \in \mathcal{T}_{\Sigma(A)} & \\
\Longleftrightarrow & \beta[t / x] \in \Gamma \text { for some } t \in \mathcal{T}_{\Sigma(A)} & \text { Inductive hypothesis } \\
\Longleftrightarrow & \exists x . \beta \in \Gamma \text { for some } t \in \mathcal{T}_{\Sigma(A)} & \text { Existence property }
\end{array}
$$

It is useful to interpret these results on classically-saturated theories also in terms of support semantics. To do this, we firstly introduce the concept of classical part of a theory - and for later use, also the concept of CIAnt part of a theory.

Definition 22 (Classical part and CIAnt part of $\Phi$ ) Let $\Phi$ be a theory. The classical part of $\Phi\left(\left.\Phi\right|_{C l}\right)$ and the ClAnt part of $\Phi\left(\Phi\left\lceil_{\mid \mathrm{CIAnt}}\right)\right.$, are defined as the set of classical formulas contained in $\Phi$ and the set of ClAnt formulas contained in $\Phi$ respectively.

Corollary 23 The followings hold: 
- Let $\mathcal{M}$ be a $\mathcal{T}_{\Sigma(A)}$-covered model on the signature $\Sigma(A)$ and $w$ a world of $\mathcal{M}$. Then $\left.\operatorname{Th}(\mathcal{M},\{w\})\right|_{\text {cl }}$ is a classically-saturated $\Sigma(A)$-theory.

- Let $\Gamma$ be a classically-saturated $\Sigma(A)$-theory. Then there exists a model $\mathcal{M}$ on the signature $\Sigma(A)$ and a world $w$ of $\mathcal{M}$ such that $\Gamma=\left.\operatorname{Th}(\mathcal{M},\{w\})\right|_{c l}$.

If we restrict our attention to $\mathcal{T}_{\Sigma(A)}$-covered models in the signature $\Sigma(A)$, Corollary 23 tells us that classically saturated theories are exactly the classical parts of theories of singleton information states, that is, states of the form $\{w\}$.

It is worth noticing that for an arbitrary information state $s$, the set $\left.\operatorname{Th}(\mathcal{M}, s)\right|_{c l}$ is not necessarily classically-saturated. Take for example an enhanced version of the information model $\mathcal{N}$ from Fig. 1a, obtained by

- extending the signature of $\mathcal{N}$ with the set of parameters $A$;

- interpreting the elements of $A$ so as to cover the whole domain $\{a, b, c\}$.

Let us call this enhanced model $\mathcal{N}^{\prime}$ and fix $s:=\{w, v\}$. Since $\mathcal{N}^{\prime}, s \not \models P(b)$ and $\mathcal{N}^{\prime}, s \not \models \neg P(b)$, we have that $\left.\operatorname{Th}\left(\mathcal{N}^{\prime}, s\right)\right|_{c l}$ is not complete-independently from the in terpretation of the new parameters-and consequently $\left.\operatorname{Th}\left(\mathcal{N}^{\prime}, s\right)\right|_{c l}$ does not satisfy the Classical disjunction property.

Finally, we restrict our attention only to CIAnt formulas, obtaining the concept of ClAnt-saturated theory.

Definition 24 (ClAnt-theories and ClAnt-saturated theories) A ClAnt $\Sigma$-theory is a $\Sigma$-theory containing only $\mathrm{ClAnt}$ formulas.

We say that a ClAnt $\Sigma(A)$-theory $\Phi$ is ClAnt-saturated (with respect to $A$ ) if for every ClAnt sentences $\varphi, \psi$ of $\Sigma(A)$ it satisfies:

- Coherence: $\quad \Phi \nvdash \perp$;

- Deductive closure: $\quad$ If $\Phi \sim \varphi$, then $\varphi \in \Phi$;

- Disjunction property: If $\Phi \sim \varphi \mathbb{\psi} \psi$ then $\Phi \vdash \varphi$ or $\Phi \vdash \psi$;

- Existence property: If $\Phi \vdash \bar{\exists} x . \varphi$ then $\Phi \vdash \varphi[t / x]$ for some $t \in \mathcal{T}_{\Sigma(A)}$;

- Normality condition: If $\Phi \not \psi \forall x . \varphi$ then $\Phi \not \psi \varphi[t / x]$ for some $t \in \mathcal{T}_{\Sigma(A)}$.

From Definition 14 it follows readily that, given $\Phi$ a saturated $\Sigma(A)$-theory, the subset of its ClAnt formulas is a ClAnt-saturated $\Sigma(A)$-theory. The converse-that every ClAnt-saturated theory can be obtained by restricting a saturated theory in the whole language - is not as obvious, but surprisingly it is the case.

Theorem 25 Let $\Phi$ be a ClAnt-saturated $\Sigma(A)$-theory. Then there exists a saturated $\Sigma(A)$-theory $\Psi$ such that $\Phi=\left.\Psi\right|_{\text {CIAnt }}$.

The rest of this section is dedicated to proving this result. Henceforth, given a ClAnttheory $\Phi$, we will indicate with $\bar{\Phi}$ its deductive closure with respect to - -which is again a ClAnt-theory.

Lemma 26 Given $\Phi$ a ClAnt-saturated $\Sigma(A)$-theory and $\alpha$ a classical sentence such that $\Phi \nvdash \neg \alpha$, then $\overline{\Phi \cup\{\alpha\}}$ is ClAnt-saturated $\Sigma(A)$-theory.

Proof Let us call $\Theta:=\overline{\Phi \cup\{\alpha\}}$. Clearly $\Theta$ is deductively closed. Moreover it is coherent, since $\Phi \nvdash \neg \alpha$ iff $\Phi, \alpha \nvdash \perp$ : 
the left-to-right implication can be deduced using the rule $(\mathrm{ClAnt} \rightarrow i)$; the rightto-left implication can be deduced using the rule $(\rightarrow e)$.

So we just need to show that $\Theta$ satisfies the disjunction property, the existence property and the normality condition.

\section{Disjunction property}

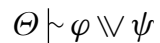

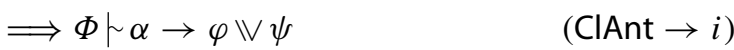

$$
\begin{aligned}
& \Longrightarrow \Phi \vdash(\alpha \rightarrow \varphi) \mathbb{\vee}(\alpha \rightarrow \psi) \quad(\mathbb{V}-\text { split }) \\
& \Longrightarrow \Phi \vdash \alpha \rightarrow \varphi \text { or } \Phi \vdash \alpha \rightarrow \psi \quad \text { Disjunction property of } \Phi \\
& \Longrightarrow \Theta \vdash \varphi \text { or } \Theta \vdash \psi \quad(\rightarrow e)
\end{aligned}
$$

\section{Existence property}

Notice that since $\alpha$ is a sentence, $x$ does not appear free in $\alpha$.

$$
\begin{array}{rlrl} 
& \Theta \vdash \bar{\exists} x . \varphi & \\
\Longrightarrow & \Phi \vdash \alpha \rightarrow \bar{\exists} x . \varphi & & (\rightarrow i) \\
\Longrightarrow & \Phi \vdash \bar{\exists} x .(\alpha \rightarrow \varphi) & & (\bar{\exists}-\text { split }) \\
\Longrightarrow & \Phi \vdash \alpha \rightarrow \varphi[t / x] \text { for some } t \in \mathcal{T}_{\Sigma(A)} & & \text { Existence property of } \Phi \\
\Longrightarrow & \Theta \vdash \varphi[t / x] \text { for some } t \in \mathcal{T}_{\Sigma(A)} & & (\rightarrow e)
\end{array}
$$

\section{Normality condition}

Notice that since $\alpha$ is a sentence and $\Theta$ is a theory, $x$ and $y$ do not appear free in $\alpha$ nor $\Theta$.

$$
\begin{aligned}
& \Theta \vdash \varphi[t / x] \text { for all } t \in \mathcal{T}_{\Sigma(A)} & & \\
\Longrightarrow & \Phi \vdash \alpha \rightarrow \varphi[t / x] \text { for all } t \in \mathcal{T}_{\Sigma(A)} & & (\text { ClAnt } \rightarrow i) \\
\Longrightarrow & \Phi \vdash \forall x .(\alpha \rightarrow \varphi) & & \text { Normality condition of } \Phi \\
\Longrightarrow & \Phi \vdash \alpha \rightarrow \varphi[y / x] & & (\forall e) \\
\Longrightarrow & \Theta \vdash \varphi[y / x] & & (\rightarrow e) \\
\Longrightarrow & \Theta \vdash \forall x . \varphi & & (\forall i)
\end{aligned}
$$

Lemma 27 Let $\Phi, \Psi$ be two ClAnt-saturated $\Sigma(A)$-theories such that $\Phi \uparrow_{c l}=\Psi \uparrow_{c l}$. Then $\Phi=\Psi$.

Proof We start with a sub-lemma: given any $\Phi$ and $\Psi$ as in the hypothesis, then for every classical formula $\alpha$ it holds $\left.(\overline{\Phi \cup\{\alpha\}})\right|_{c l}=\left.(\overline{\Psi \cup\{\alpha\}})\right|_{c l}$. The proof is 
straightforward:

$$
\begin{array}{rlrl} 
& \beta \in \overline{\Phi \cup\{\alpha\}} & \\
\Longrightarrow & \alpha \rightarrow \beta \in \Phi & (\rightarrow i) \\
\Longrightarrow & \alpha \rightarrow \beta \in \Psi & & \text { Assumption } \\
\Longrightarrow & \beta \in \overline{\Psi \cup\{\alpha\}} & (\rightarrow e)
\end{array}
$$

Using this technical result, we can show by induction on the length of the CIAnt sentence $\theta$-intended as the number of symbols appearing in $\theta$ - that $\theta \in \Phi \Longleftrightarrow$ $\theta \in \Psi$.

- If $\theta$ is an atom, the result follows by hypothesis_-atoms are classical formulas.

- If $\theta \equiv \psi \wedge \chi$, then

$$
\begin{aligned}
& \theta \in \Phi \\
\Longleftrightarrow \psi \in \Phi \text { and } \chi \in \Phi & \text { Deductive closure of } \Phi \\
\Longleftrightarrow \psi \in \Psi \text { and } \chi \in \Psi & \text { Inductive hypothesis } \\
\Longleftrightarrow \theta \in \Psi & \text { Deductive closure of } \Psi
\end{aligned}
$$

- If $\theta \equiv \psi \vee \chi$, then

$$
\begin{aligned}
\theta \in \Phi & \\
\Longleftrightarrow \psi \in \Phi \text { or } \chi \in \Phi & \text { Deductive closure and Disjunction property of } \Phi \\
\Longleftrightarrow \psi \in \Psi \text { or } \chi \in \Psi & \text { Inductive hypothesis } \\
\Longleftrightarrow \theta \in \Psi & \text { Deductive closure and Disjunction property of } \Psi
\end{aligned}
$$

- If $\theta \equiv \alpha \rightarrow \psi$, then

$$
\begin{aligned}
& \theta \in \Phi \\
\Longleftrightarrow \psi \in \overline{\Phi \cup\{\alpha\}} & (\text { ClAnt } \rightarrow i) \text { and }(\rightarrow e) \\
\Longleftrightarrow \psi \in \overline{\Psi \cup\{\alpha\}} & \text { Inductive hypothesis applied to } \overline{\Phi \cup\{\alpha\}} \text { and } \overline{\Psi \cup\{\alpha\}} \\
\Longleftrightarrow \theta \in \Psi & (\text { ClAnt } \rightarrow i) \text { and }(\rightarrow e)
\end{aligned}
$$

Notice that we can apply the inductive hypothesis to the theories $\overline{\Phi \cup\{\alpha\}}$ and $\overline{\Psi \cup\{\alpha\}}$ since by Lemma 26 they are CIAnt-saturated theories, and we showed at the beginning of the proof that $\left.\overline{\Phi \cup\{\alpha\}}\right|_{c l}=\left.\overline{\Psi \cup\{\alpha\}}\right|_{c l}$.

- If $\theta \equiv \bar{\exists} x . \psi$, then

$$
\begin{array}{cll} 
& \theta \in \Phi & \\
\Longleftrightarrow \psi[t / x] \in \Phi & \text { for some } t \in \mathcal{T}_{\Sigma(A)} & \text { Existence property for } \Phi \\
\Longleftrightarrow \psi[t / x] \in \Psi & \text { for some } t \in \mathcal{T}_{\Sigma(A)} & \text { Inductive hypothesis } \\
\Longleftrightarrow \theta \in \Psi & & \text { Existence property for } \Psi
\end{array}
$$


- If $\theta \equiv \forall x . \psi$, then

$$
\begin{array}{rll} 
& \theta \in \Phi & \\
\Longleftrightarrow \varphi[t / x] \in \Phi & \text { for all } t \in \mathcal{T}_{\Sigma(A)} & \text { Normality condition for } \Phi \\
\Longleftrightarrow \psi[t / x] \in \Psi & \text { for all } t \in \mathcal{T}_{\Sigma(A)} & \text { Inductive hypothesis } \\
\Longleftrightarrow \theta \in \Psi & & \text { Normality condition for } \Psi
\end{array}
$$

To obtain a result analogous to Corollary 23, we need to introduce a construction resembling the canonical models for intuitionistic logic.

Definition 28 (Canonical model) We define $\mathcal{M}^{c}$ the canonical model of $\Sigma(A)$ by the following clauses:

- The set of worlds is $W^{c}$, the set of classically-saturated $\Sigma(A)$-theories;

- The common domain of the structures is $D^{c}:=\mathcal{T}_{\Sigma(A)}$;

- The model corresponding to world $\Gamma$ is $M_{\Gamma}$-introduced in Definition 20.

As a direct consequence of Definition 28 and of Lemma 21, we have that $\left.\operatorname{Th}\left(\mathcal{M}^{c},\{\Gamma\}\right)\right|_{c l}=\Gamma$ for every $\Gamma \in W^{c}$. From this observation we obtain the following Lemma.

Lemma 29 Let $s \subseteq W^{c}$ and $\Gamma$ a classical theory. Then

$$
\mathcal{M}^{c}, s \vDash \Gamma \Longleftrightarrow \forall \Theta \in s . \Gamma \subseteq \Theta
$$

Proof For every $\alpha \in \Gamma$ we have

$$
\begin{aligned}
\mathcal{M}^{c}, s \vDash \alpha & \Longleftrightarrow \forall \Theta \in s . \alpha \in \operatorname{Th}\left(\mathcal{M}^{c},\{\Theta\}\right) \quad \text { (By Lemma 8) } \\
& \Longleftrightarrow \forall \Theta \in s . \alpha \in \Theta
\end{aligned}
$$

Given a coherent classical $\Sigma(A)$-theory $\Theta$ it is not generally true that there exists a world of the canonical model satisfying $\Theta$. A simple example, for $P$ a unary predicate symbol, is $\Theta:=\{\neg \forall x . P(x)\} \cup\left\{P(t) \mid t \in \mathcal{T}_{\Sigma(A)}\right\}$. The problem in this case is that for every theory $\Gamma$ of a world of the canonical model — that is, by Lemma 29, for every classically-saturated $\Sigma(A)$-theory $\Gamma$-if $\Gamma \forall \forall x . P(x)$ then there must be a witness $t \in \mathcal{T}_{\Sigma(A)}$ for which $\Gamma \mid \forall P(t)$.

So the normality condition is necessary for such a world to exist. The following lemma shows that it is also a sufficient condition. ${ }^{5}$

Lemma 30 (Classical saturation lemma) Let $\Theta$ be a coherent classical $\Sigma(A)$-theory such that for every sentence $\alpha$ in the signature $\Sigma(A)$ it holds:

$$
\text { Normality condition: } \quad \Theta \forall \forall x . \alpha \Longrightarrow \Theta \forall \alpha[t / x] \text {, for some } t \in \mathcal{T}_{\Sigma(A)} \text {. }
$$

\footnotetext{
5 Recall that, by Lemma 10, $\vdash$ amounts to classical entailment when restricted to classical formulas.
} 
Then there exists a classically-saturated $\Sigma(A)$-theory $\Gamma$ such that $\Theta \subseteq \Gamma$.

Proof This proof is an adaptation of the proof of Theorem (Gabbay 1981, Sec. 3.3, Theorem 2) for the intuitionistic case.

We start by showing a useful property, that we will later refer to as $(*)$-property: given $\beta$ a classical sentence of $\Sigma(A)$, if $\Delta$ is a classical theory that satisfies the normality condition above then $\Delta \cup\{\beta\}$ also satisfies the condition. In fact for any classical formula $\alpha$ we have

$$
\begin{aligned}
\Delta \cup\{\beta\} \vdash \forall x . \alpha & \Longrightarrow \Delta \vdash \beta \rightarrow \forall x . \alpha \\
& \Longrightarrow \Delta \vdash \forall x .(\beta \rightarrow \alpha) \\
& \Longrightarrow \Delta \vdash \beta \rightarrow \alpha[t / x] \text { for some } t \in \mathcal{T}_{\Sigma(A)} \\
& \Longrightarrow \Delta \cup\{\beta\} \vdash \alpha[t / x] \text { for some } t \in \mathcal{T}_{\Sigma(A)}
\end{aligned}
$$

Now we go back to the main proof. Fix an enumeration $B_{0}, B_{1}, \ldots$ of the classical sentences in the signature $\Sigma(A)$. We will define inductively a chain of classical $\Sigma(A)$ theories $\Gamma_{i}$ indexed by $\mathbb{N}$ such that:

1. $\Gamma_{i}$ is coherent, that is, $\Gamma_{i} \mid+\perp$.

2. For every index $i, \Gamma_{i} \subseteq \Gamma_{i+1}$.

3. For every index $i, \Gamma_{i}$ respects the normality condition.

The plan is to take $\Gamma:=\cup_{i \in \mathbb{N}} \Gamma_{i}$. During the construction we will impose some additional conditions to ensure $\Gamma$ to be a classically-saturated $\Sigma(A)$-theory. We start the construction by defining $\Gamma_{0}:=\Theta$. By hypothesis conditions 1 and 3 are respected; condition 2 is trivially respected.

Suppose we already defined $\Gamma_{n}$ with the properties above. We proceed by cases.

- Case $\Gamma_{n} \nmid \neg B_{n}$ and $B_{n} \neq \exists x . \alpha$. Define $\Gamma_{n+1}:=\Gamma_{n} \cup\left\{B_{n}\right\}$. Condition 1 follows from $\Gamma_{n} \nmid \neg B_{n}$; condition 2 is trivially satisfied; condition 3 follows from the (*)-property.

- Case $\Gamma_{n} \not \nvdash \neg B_{n}$ and $B_{n}=\exists x . \alpha$. Notice that $\Gamma_{n} \cup\{\exists x . \alpha\} \mid \forall \forall x . \neg \alpha$. So by condition 3 and the (*)-property, there exists a term $t \in \mathcal{T}_{\Sigma(A)}$ such that $\Gamma_{n} \cup\{\exists x . \alpha\} \mid \neg \neg \alpha[t / x]$. Define $\Gamma_{n+1}:=\Gamma_{n} \cup\left\{B_{n}, \alpha[t / x]\right\}$. Condition 1 follows from $\Gamma_{n} \mid \forall \neg B_{n}$ and $\Gamma_{n} \cup$ $\left\{B_{n}\right\} \mid \forall \neg \alpha[t / x]$; Condition 2 trivially holds; Condition 3 follows from the $(*)$ property.

- Case $\Gamma_{n} \vdash \neg B_{n}$. Define $\Gamma_{n+1}:=\Gamma_{n}$. Conditions 1,2 and 3 trivially hold.

Define $\Gamma:=\cup_{i \in \mathbb{N}} \Gamma_{i}$. By Condition $2, \Theta \subseteq \Gamma$. So it remains to show that $\Gamma$ is classically-saturated. First of all, $\Gamma$ is coherent since $\Gamma \vdash \perp$ iff there exists an index $i \in \mathbb{N}$ such that $\Gamma_{i} \vdash \perp$, but the latter would contradict condition 1 . Moreover $\Gamma$ is deductively closed, since if $\Gamma \vdash B_{n}$ for some $n \in \mathbb{N}$, then $\Gamma_{n} \not \vdash \neg B_{n}$ and so $B_{n} \in$ $\Gamma_{n+1} \subseteq \Gamma$.

To show the classical disjunction property, suppose that $\Gamma \vdash B_{m} \vee B_{n}$. This implies that $\Gamma \mid \forall \neg B_{m}$ or $\Gamma \mid \forall \neg B_{n}$; without loss of generality, suppose the former is the case. Then $\Gamma_{m} \nvdash \neg B_{m}$, and by construction $B_{m} \in \Gamma_{m+1} \subseteq \Gamma$. Finally, to show the classical existence property suppose that $\Gamma \vdash \exists x . \alpha$ and let $B_{n}$ be the enumeration of $\exists x . \alpha$. 
Then $\Gamma \mid \forall \neg \exists x . \alpha$, and consequently $\Gamma_{n} \nvdash \neg \exists x . \alpha$. By construction, there exists a term $t \in \mathcal{T}_{\Sigma(A)}$ such that $\alpha[t / x] \in \Gamma_{n+1} \subseteq \Gamma$.

This shows that $\Gamma$ is a classically-saturated $\Sigma(A)$-theory as wanted.

Combining the results above, we can show the connection between CIAnt-saturated theories and the semantics of the logic.

Theorem 31 Given $\Phi$ a ClAnt-saturated $\Sigma(A)$-theory, there exists a state $E_{\Phi}$ of $\mathcal{M}^{c}$ such that for every CIAnt formula $\psi$ of $\Sigma(A)$ :

$$
\psi \in \Phi \Longleftrightarrow \mathcal{M}^{c}, E_{\Phi} \vDash \psi
$$

Proof Define the state $E_{\Phi}:=\left\{\Gamma \in W^{c}|\Phi|_{c l} \subseteq \Gamma\right\}$ and consider the theory of this state $\Psi:=\operatorname{Th}\left(\mathcal{M}^{c}, E_{\Phi}\right)$. By Lemma $29,\left.\Phi\right|_{c l} \subseteq \Psi \uparrow_{c l}$. We want to show that also the other inclusion holds.

Fix a classical formula $\alpha \in \Psi$ and suppose toward a contradiction that $\left.\alpha \notin \Phi\right|_{c l}$. In particular, $\alpha$ is not a consequence of $\left.\Phi\right|_{c l}$ in classical first-order logic-since $\left.\Phi\right|_{c l}$ is classically-saturated. By Lemma 30 , then there exists a classically-saturated $\Sigma(A)$ theory $\Theta$ such that $\left.\Phi\right|_{c l} \subseteq \Theta$ and $\alpha \notin \Theta$. But this leads to a contradiction, since we have:

$$
\begin{aligned}
\alpha \in \Psi & \Longrightarrow \forall \Gamma \in E_{\Phi} \cdot \alpha \in \Gamma \quad \text { (by Lemma 29) } \\
& \left.\Longrightarrow \forall \Gamma \supseteq \Phi\right|_{c l} \cdot \alpha \in \Gamma \quad
\end{aligned}
$$

So we established that $\left.\alpha \in \Phi\right|_{c l}$, and since $\alpha$ was an arbitrary classical formula in $\Psi$, we also established that $\Psi \uparrow_{c l} \subseteq \Psi \uparrow_{c l}$.

By Lemma 27, since $\Phi \uparrow_{c l}=\Psi \uparrow_{c l}$, we obtain that $\Phi=\Psi \uparrow_{\text {CIAnt }}$, from which the result follows.

From the previous result, Theorem 25 follows trivially.

Proof (Theorem 25) Consider $\Psi:=\operatorname{Th}\left(\mathcal{M}^{c}, E_{\Phi}\right)$. Since $\mathcal{M}^{c}$ is $\mathcal{T}_{\Sigma(A)}$-covered, $\Psi$ is a saturated $\Sigma(A)$-theory. Moreover, by Theorem 31, $\Phi=\Psi \uparrow_{\text {CIAnt }}$, as wanted.

This result, together with the saturation lemma presented in Sect. 5, leads to the completeness of the natural deduction system introduced for CIAnt.

\section{Completeness}

This section is completely devoted to the proof of completeness for the CIAnt fragment. To lighten the notation in the proofs of the following lemmas, we introduce the following convention for inferences with multiple conclusions: let $\Phi$ and $\Psi$ be sets of ClAnt formulas; we write $\Phi \vdash \Psi$ to indicate that there exists $\psi_{1}, \ldots, \psi_{n} \in \Psi$ such that $\Phi \vdash \psi_{1} \vee \ldots \vee \vee \psi_{n}$ or, in case $\Psi$ is empty, that $\Phi \vdash \perp$.

Lemma 32 Let $\Phi \cup \Psi \cup\{\chi\}$ be a set of CIAnt formulas. If $\Phi \cup\{\chi\} \vdash \Psi$ and $\Phi \vdash \Psi \cup\{\chi\}$, then $\Phi \vdash \Psi$. 
Proof By hypothesis, for some $\varphi_{i}, \varphi_{i^{\prime}}^{\prime} \in \Phi$ and $\psi_{j}, \psi_{j^{\prime}}^{\prime} \in \Psi$, we have 6

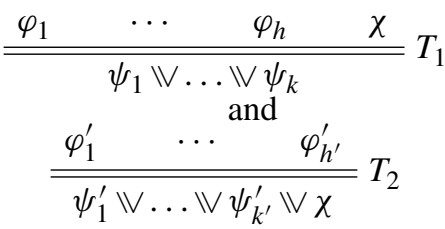

Combining the two proofs together we get

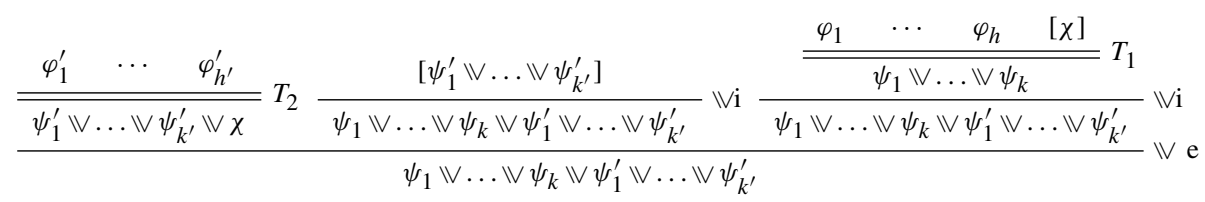

and thus $\Phi \vdash \Psi$, as wanted.

Lemma 33 (Saturation lemma) Consider $\Phi \cup\{\psi\}$ a set of CIAnt $\Sigma$-formulas such that $\Phi \nvdash \psi$. Consider the objects $\widetilde{V}, \widetilde{\Phi}$ and $\widetilde{\psi}$ as defined in Definition 16. Then given A a countable set of parameters disjoint from $\Sigma(\widetilde{V})$, there exists a ClAnt-saturated $\Sigma(A \cup \widetilde{V})$-theory $\Delta$ such that $\widetilde{\Phi} \subseteq \Delta$ and $\widetilde{\psi} \notin \Delta$.

Proof This proof is an adaptation of the proof of Theorem 2 in Gabbay (1981, Sec. 3.3), developed for intuitionistic logic.

First of all, by Proposition 17 we can assume that $\Phi \cup\{\psi\}$ contains only sentences, and that we just need to find a CIAnt-saturated $\Sigma(A)$-theory $\Delta$ such that $\Phi \subseteq \Delta$ and $\psi \notin \Delta$. Fix an enumeration $B_{1}, B_{2}, \ldots$ of the ClAnt sentences in the signature $\Sigma(A) .^{7}$ We will define inductively a chain of pairs of $\Sigma(A)$-theories $\left\langle\Delta_{i}, \Theta_{i}\right\rangle$ indexed by $i \in \mathbb{N}$ such that:

1. $\Delta_{i} \not \Theta_{i}$.

2. For every index $i, \Delta_{i} \subseteq \Delta_{i+1}$ and $\Theta_{i} \subseteq \Theta_{i+1}$.

3. $B_{n} \in \Delta_{n+1} \cup \Theta_{n+1}$.

The plan is to take $\Delta:=\cup_{i \in \mathbb{N}} \Delta_{i}$. During the construction we will impose some additional conditions to ensure $\Delta$ to be a CIAnt-saturated $\Sigma(A)$-theory.

We start the construction by defining $\left\langle\Delta_{0}, \Theta_{0}\right\rangle:=\langle\Phi,\{\psi\}\rangle$. By hypothesis condition 1 is respected; conditions 2 and 3 are trivially respected.

Suppose we already defined $\left\langle\Delta_{n}, \Theta_{n}\right\rangle$ with the properties above.

By Lemma 32, we cannot have both that $\Delta_{n} \sim \Theta_{n} \cup\left\{B_{n}\right\}$ and that $\Delta_{n} \cup\left\{B_{n}\right\} \sim \Theta_{n}$. So we continue the proof by considering two possible (non mutually exclusive) cases: if $\Delta_{n} \not \psi \Theta_{n} \cup\left\{B_{n}\right\}$ and if $\Delta_{n} \cup\left\{B_{n}\right\} \mid \psi \Theta_{n}$.

\footnotetext{
${ }^{6}$ In the natural deduction proofs that follow we will use a single line for the application of an instance of a rule; while we will use a double line for a subproof. On the right of a single line we will write the name of the rule applied; on the right of a double line we will write a label naming the subproof.

${ }^{7}$ Notice that this can be done without the use of the axiom of choice since we are considering a countable signature $\Sigma$ and a countable set $A$.
} 
1. Case $\Delta_{n} \not \psi \Theta_{n} \cup\left\{B_{n}\right\}$. We distinguish two sub-cases, depending on whether $B_{n}$ is of the form $\forall x . \varphi$ or not.

(a) Case $B_{n}=\forall x . \varphi$. Consider a fresh parameter $a \in A$ (that is, an element not appearing in $\left.\Delta_{n} \cup \Theta_{n} \cup\left\{B_{n}\right\}\right)$ and define $\Delta_{n+1}:=\Delta_{n}$ and $\Theta_{n+1}:=\Delta_{n} \cup$ $\left\{B_{n}, \varphi[a / x]\right\}$.

Clearly conditions 2 and 3 are respected. Moreover also condition 1 holds, i.e. $\Delta_{n+1} \nvdash \Theta_{n+1}$; for otherwise, for some $\delta_{1}, \ldots, \delta_{h} \in \Delta_{n}$ and some $\theta_{1}, \ldots, \theta_{k} \in$ $\Theta_{n}$, we would have:

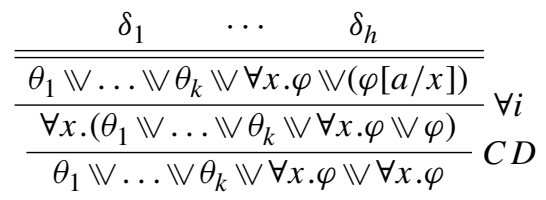

So in particular $\Delta_{n} \sim \Theta_{n} \cup\left\{B_{n}\right\}$, which is a contradiction.

(b) Case $B_{n} \neq \forall x . \varphi$. In this case we simply define $\Delta_{n+1}:=\Delta_{n}$ and $\Theta_{n+1}:=\Theta_{n} \cup$ $\left\{B_{n}\right\}$. Conditions 1,2 and 3 follow by construction.

2. Case $\Delta_{n} \cup\left\{B_{n}\right\} \not \Theta_{n}$. Once again, we distinguish two sub-cases, this time depending on whether $B_{n}$ is of the form $\bar{\exists} x . \varphi$ or not.

(a) Case $B_{n}=\bar{\exists} x \cdot \varphi$ Consider a fresh parameter $a \in A$ and define $\Delta_{n+1}:=\Delta_{n} \cup$ $\left\{B_{n}, \varphi[a / x]\right\}$ and $\Theta_{n+1}:=\Theta_{n}$.

Clearly conditions 2 and 3 are respected. Also condition 1 holds, for otherwise:

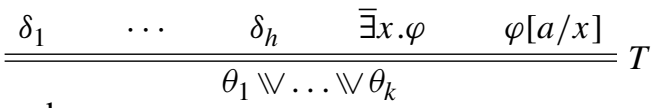

and so

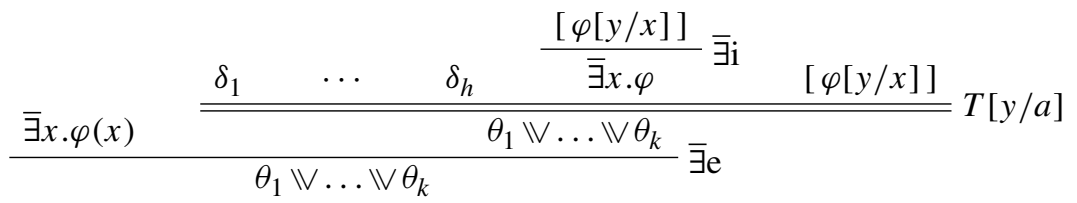

where $T[y / a]$ is the proof obtained by substituting a fresh variable $y$ for $a$ in the proof $T$. Thus $\Delta_{n} \cup\left\{B_{n}\right\} \vdash \Theta_{n}$, which is a contradiction.

(b) Case $B_{n} \neq \bar{\exists} x . \varphi$. Define $\Delta_{n+1}:=\Delta_{n} \cup\left\{B_{n}\right\}$ and $\Theta_{n+1}:=\Theta_{n}$; clearly conditions 1,2 and 3 are respected.

Thus we built the sequence $\left\langle\Delta_{i}, \Theta_{i}\right\rangle$ for $i \in \mathbb{N}$, as wanted. Define $\Delta:=\cup_{i \in \mathbb{N}} \Delta_{i}$ and $\Theta:=\cup_{i \in \mathbb{N}} \Theta_{i}$. We want to show now that $\Delta$ is ClAnt-saturated, that $\Phi \subseteq \Delta$ and that $\psi \notin \Delta$.

First of all, notice that $\Phi \subseteq \Delta_{0} \subseteq \Delta$. Moreover by condition 1 and 2 we have $\Delta \nvdash \Theta$, for otherwise there would be a finite $n$ such that $\Delta_{n} \sim \Theta_{n}$. Consequently $\psi \in \Theta_{0} \subseteq \Theta$, and so $\psi \notin \Delta$. What is left to show is that $\Delta$ is CIAnt-saturated.

By condition 3, we have that every sentence in CIAnt is an element of $\Delta \cup \Theta$. This, together with $\Delta \not \Theta$ and $\Theta \neq \emptyset$, ensures that $\Delta$ is deductively closed and $\perp \notin \Delta$. 
As for the Disjunction property, suppose $\Delta \sim \varphi \mathbb{\psi} \psi$. By contradiction, assume $\varphi, \psi \notin \Delta$, which in turn implies $\varphi, \psi \in \Theta$. In particular we would have $\Delta \sim \Theta$, which is a contradiction; thus at least one among $\varphi$ and $\psi$ has to be in $\Delta$.

For the Existence property, suppose $\bar{\exists} x . \varphi \in \Delta$. Let $B_{n}=\bar{\exists} x . \varphi$ be the enumeration given to this sentence.

We have $B_{n} \in \Delta_{n+1} \cup \Theta_{n+1}$ by condition 3. But if $B_{n} \in \Theta_{n+1}$ were the case, we would have $\Delta \sim \Theta_{n+1}$ and consequently $\Delta \vdash \Theta$, which is a contradiction. So it follows that $B_{n} \in \Delta_{n+1}$. In particular, following the inductive construction presented above (case 2a), we have that $\Delta_{n+1}:=\Delta_{n} \cup\left\{B_{n}, \varphi[a / x]\right\}$ for some $a \in A \subseteq \mathcal{T}_{\Sigma(A)}$. And so we have $\varphi[a / x] \in \Delta_{n+1} \subseteq \Delta$. Since $\bar{\exists} x . \varphi$ is an arbitrary existential sentence, it follows that $\Delta$ has the existence property.

The normality condition follows from considerations completely analogous to the one in the previous paragraph.

Using the results we collected so far, we can completely characterize the connection between the relation $\digamma$ and the relations $\vdash$ and $\vDash$.

Theorem 34 Let $\Phi \cup\{\psi\}$ be CIAnt formulas. Then it holds:

$$
\Phi \vdash \psi \quad \Longleftrightarrow \quad \Phi \vdash \psi
$$

Proof The left-to-right implication follows trivially, since every derivation in the deductive system for ClAnt is also a derivation for the deductive system for InqBQ.

For the right-to-left implication, we show the contrapositive. Suppose that $\Phi \nvdash \psi$. Then by Lemma 33 there exists a ClAnt-saturated theory $\Delta$ (in an extended signature) such that $\Phi \subseteq \Delta$ and $\psi \notin \Delta$. By Theorem 25, there exists a saturated theory $\Psi$ such that $\Delta=\Psi \uparrow_{\text {ClAnt }}$, and so in particular $\Phi \subseteq \Psi$ and $\psi \notin \Psi$. Since $\Psi$ is deductively closed with respect to $\vdash$, it follows that $\Phi \nvdash \psi$.

Theorem 35 (Completeness) Let $\Phi \cup\{\psi\}$ be CIAnt formulas. Then it holds:

$$
\Phi \vDash \psi \quad \Longrightarrow \quad \Phi \vdash \psi
$$

Proof We prove the result by contraposition: suppose that $\Phi \nvdash \psi$. By Lemma 33, given $A$ a countable set of fresh parameters there exists a CIAnt-saturated $\Sigma(\widetilde{V} \cup A)$-theory $\Delta$ such that $\widetilde{\Phi} \subseteq \Delta$ and $\widetilde{\psi} \notin \Delta$. By Theorem 31 , given $\mathcal{M}^{c}$ the canonical model for the signature $\Sigma(\widetilde{V} \cup A)$, we have $\left.\operatorname{Th}\left(\mathcal{M}^{c}, E_{\Delta}\right)\right|_{\text {CIAnt }}=\Delta$. Thus in particular $\mathcal{M}^{c}, E_{\Delta} \vDash \widetilde{\Phi}$ and $\mathcal{M}^{c}, E_{\Delta} \not \models \widetilde{\psi}$.

Define the assignment $g: V \rightarrow D$ such that $g(x)=(\widetilde{x})^{\mathcal{M}}$. An easy induction shows that, for every formula $\chi$ in the signature $\Sigma$ with free variables in $V$ we have $\mathcal{M}^{c}, E_{\Delta} \vDash_{g} \chi$ iff $\mathcal{M}^{c}, E_{\Delta} \vDash \tilde{\chi}$. In particular, it follows that $\mathcal{M}^{c}, E_{\Delta} \vDash_{g} \Phi$ and $\mathcal{M}^{c}, E_{\Delta} \not \nvdash_{g} \psi$. Thus $\Phi \not \models \psi$, as wanted.

\section{Language with Equality}

Notice that in defining the syntax of InqBQ (Definition 1) we did not consider the equality symbol. There are at least two ways to enhance the semantics of InqBQ with 
an equality symbol: we can consider a rigid or a non-rigid interpretation. Both are described in detail in Ciardelli (2016, Ch. 4). ${ }^{8}$

In the rigid interpretation, the semantic clause for equality is

$$
\mathcal{M}, s \vDash_{g} t_{1}=t_{2} \quad \Longleftrightarrow \quad t_{1}^{g}=t_{2}^{g} .
$$

That is, $t_{1}=t_{2}$ holds if and only if the two elements are interpreted as the same element of $D^{\mathcal{M}}$.

In the second case, we want the interpretation of equality to be world-dependent, like the interpretation of other relation symbols. To achieve this, we need a small generalization of the notion of first-order model described in Footnote 3: additionally, we require a first-order model to specify an equivalence relation $\sim$ (the interpretation of the equality symbol) that is required to be a congruence with respect to the interpretation of the function and relation symbols. In this case, the corresponding semantical clause for equality is

$$
\mathcal{M}, s \vDash_{g} t_{1}=t_{2} \quad \Longleftrightarrow \quad \forall w \in s . t_{1}^{g} \sim_{w} t_{2}^{g} .
$$

That is, $t_{1}$ and $t_{2}$ are interpreted as elements of $D^{\mathcal{M}}$ which every world in s consider to be identical.

Let us refer to $\models^{=}$and $\models^{\sim}$ as the semantical relations corresponding to Clauses 1 and 2 respectively. To account for these two extensions of the language, we can modify the deductive system in Definition 9 by adding rules 1-3 for the rigid equality; and rules $1-2$ for the non-rigid equality. ${ }^{9}$

$$
1 \bar{t}_{t=t} \quad 2 \frac{\varphi[t / x] \quad t=t^{\prime}}{\varphi\left[t^{\prime} / x\right]} \quad 3 \frac{}{\forall x, y . ? x=y}
$$

As for the case without equality, it is still an open question whether these deductive systems are complete. However, we can show that the corresponding restricted systems for ClAnt are complete.

Theorem 36 (Completeness for the language with equality) Consider the natural deduction systems $\mathcal{\sim}^{=}$and $\sim^{\sim}$ obtained by adding to the system in Definition 14 Rules $1-3$ and Rules $1-2$ respectively. Then, given $\Phi \cup\{\psi\}$ a set of CIAnt formulas (possibly containing $=$ ), we have:

$$
\Phi \vDash=\Longrightarrow \Phi \vdash^{=} \psi \quad \Phi \vDash^{\sim} \psi \Longrightarrow \Phi \vdash^{\sim} \psi
$$

Proof (Sketch) We want to prove analogues of Theorem 35 for the new relations introduced. To achieve this, we need analogues of Definition 20 and Lemma 21 for

\footnotetext{
8 In Ciardelli (2016), the standard interpretation of equality is the non-rigid one, defined in Ciardelli (2016, Definition 4.1.5). The rigid interpretation (called id-entailment) is described in Ciardelli (2016, Definition 4.5.2).

9 Rules 1-2 are the standard rules for equality found in natural deduction systems for intuitionistic logic, while Rule 3 is thoroughly discussed in Ciardelli (2016, Sec. 4.5).
} 
the case with rigid and non-rigid equality, which follow again by adapting the proof of Theorem 2.3.3 in Hodges (1993). All the other proofs in this paper are independent from the equality symbols being in the language, including the proof of Theorem 35 .

\section{Conclusions}

In this paper we introduced the ClAnt fragment of InqBQ, extending the mentionsome and mention-all fragments. We presented a natural deduction system for ClAnt, specializing the one proposed in Ciardelli (2016, Ch. 4) for InqBQ. We introduced CIAnt-saturated theories, which stem from the deduction system presented, and showed (1) these theories are characterized by their classical fragment and (2) they are exactly the restrictions to the CIAnt fragment of saturated theories for the whole language. This allowed us to prove the main result of the paper, that is, the completeness of the natural deduction system through a canonical model construction.

The protagonists in the proof abovementioned are the CIAnt-saturated theories: by studying their properties, we were able to adapt the canonical model technique to (a fragment of) InqBQ. The same approach can be applied to fragments of the logic satisfying properties (1) and (2) above, obtaining analogous completeness results; as of now it is not known if a proper extension of $\mathrm{ClAnt}$ with said properties exists. Potentially, a variation of the approach could be applied to the $\operatorname{logic} \operatorname{InqB} Q$, although it is not known whether property (1) holds for the whole language. Further investigations in this direction are left for future work.

Funding Open Access funding enabled and organized by Projekt DEAL.

Open Access This article is licensed under a Creative Commons Attribution 4.0 International License, which permits use, sharing, adaptation, distribution and reproduction in any medium or format, as long as you give appropriate credit to the original author(s) and the source, provide a link to the Creative Commons licence, and indicate if changes were made. The images or other third party material in this article are included in the article's Creative Commons licence, unless indicated otherwise in a credit line to the material. If material is not included in the article's Creative Commons licence and your intended use is not permitted by statutory regulation or exceeds the permitted use, you will need to obtain permission directly from the copyright holder. To view a copy of this licence, visit http://creativecommons.org/licenses/by/4.0/.

\section{References}

Ciardelli, I. (2009). Inquisitive semantics and intermediate logics. M.Sc. thesis, University of Amsterdam. Ciardelli, I. (2014). Modalities in the realm of questions: Axiomatizing inquisitive epistemic logic. In R. Goré, B. Kooi, \& A. Kurucz (Eds.), Advances in Modal Logic (AiML) (pp. 94-113). College Publications.

Ciardelli, I. (2016). Questions in logic. Institute for logic, language and computation. Ph.D. thesis, University of Amsterdam.

Ciardelli, I., \& Roelofsen, F. (2011). Inquisitive logic. Journal of Philosophical Logic, 40(1), 55-94.

Ciardelli, I., Groenendijk, J., \& Roelofsen, F. (2018). Inquisitive semantics. Oxford University Press.

Ciardelli, I., Iemhoff, R., \& Yang, F. (2020). Questions and dependency in intuitionistic logic. Notre Dame Journal of Formal Logic, 61(1), 75-115, 1.

Gabbay, D. (1981). Semantical investigations in Heyting's intuitionistic logic (Vol. 148). Springer. 
Gianluca, G., \& Ivano, C. (2019). An Ehrenfeucht-Fraïssé game for inquisitive first-order logic. In A. Silva, S. Staton, P. Sutton, \& C. Umbach (Eds.), Language, logic, and computation (pp. 166-186). Springer.

Grilletti, G. (2019). Disjunction and existence properties in inquisitive first-order logic. Studia Logica, 107(6), 1199-1234.

Groenendijk, J. (1999). The logic of interrogation. In T. Matthews \& D. Strolovitch (Eds.), Semantics and linguistic theory (pp. 109-126). Cornell University Press.

Hodges, W. (1993). Model theory. Encyclopedia of mathematics and its applications: Cambridge University Press.

Katsuhiko, S. (2011). First-order inquisitive pair logic. In M. Banerjee \& A. Seth (Eds.), Logic and its applications (pp. 147-161). Springer.

Punčochář, V. (2015). Weak negation in inquisitive semantics. Journal of Logic, Language, and Information, 24(3), 323-355.

Punčocháŕ, V. (2019). Substructural inquisitive logics. The Review of Symbolic Logic, 12(2), 296-330.

Roelofsen, F. (2013). Algebraic foundations for the semantic treatment of inquisitive content. Synthese, 190(1), 79-102.

ten Cate, B., \& Shan, C.-C. (2007). Axiomatizing Groenendijks logic of interrogation. In M. Aloni, A. Butler, \& P. Dekker (Eds.), Questions in dynamic semantics (pp. 63-82). Elsevier.

Publisher's Note Springer Nature remains neutral with regard to jurisdictional claims in published maps and institutional affiliations. 